\title{
Combined Carotid Endarterectomy and Coronary Bypass Grafting: Risk versus Benefit- A Review
}

\author{
Rezwanul Hoque1, Sabrina Sharmeen Husain ${ }^{1}$, Zerzina Rahman², Ashia Ali², \\ Mostafa Nuruzzaman², Md. Alauddin'1. \\ ${ }^{1}$ Department of Cardiac Surgery, Bangabandhu Sheikh Mujib Medical University, Dhaka. \\ ${ }^{2}$ Department of Cardiac Anesthesiology, Bangabandhu Sheikh Mujib Medical University, Dhaka. \\ Address for correspondence \\ Dr. Md. Rezwanul Hoque Bulbul \\ Associate Professor (Cardiac Surgery), Bangabandhu Sheikh Mujib Medical University, Shahbag, Dhaka, Bangladesh \\ e-mail : drrhbulbul@yahoo.com
}

\begin{abstract}
Carotid Endarterectomy (CEA) performed in combination with coronary artery bypass grafting (CABG) have also increased steadily since Bernhard and colleague's initial report in 1972. Coexistence of symptomatic coronary artery disease and significant carotid artery stenosis ranges from $3.4 \%$ to $22 \%$. The incidence of postoperative stroke after CABG ranges from $0.7 \%$ to $5 \%$. Coronary revascularization in a patient with internal carotid artery stenosis more than $50 \%$ is associated with a postoperative stroke rate of $6 \%$, which increases significantly to more than $16 \%$ when stenosis is more than $90 \%$. To reduce the potential risk for postoperative stroke after $\mathrm{CABG}$ in patients with significant or symptomatic carotid artery stenosis, many surgeons have advocated combined CABG with unilateral carotid endarterectomy. However, clinical experience with the concomitant approach is conflicting. On the basis of the long-term results, it is estimated that simultaneous carotid endarterectomy and myocardial revascularization in conjunction with cardiopulmonary bypass is a method safe enough to prefer its routine use with acceptable low operative risk and satisfactory long-term morbidity. The overall 30-day mortality of combined CABG with bilateral carotid endarterectomy was $6.1 \%$ and that was unrelated to primary cardiac or cerebrovascular events. Favorable outcome also supports the justification for performing concomitant coronary artery bypass grafting with bilateral carotid endarterectomies in selected patients.
\end{abstract}

Key words : Carotid endarterectomy, Coronary Bypass Grafting.

\section{Introduction}

Carotid endarterectomy (CEA) is a surgical procedure that was introduced in the early 1950s and is performed in both symptomatic and asymptomatic patients. The safety and efficacy of carotid endarterectomy as a strategy for stroke prevention has long been debated and given rise to unresolved controversy. In response to genuine concerns expressed by many investigators, considerable effort has resulted in publication of retrospective reviews, natural history studies, audits of community practice, position papers by various societies, expert opinion statements, and finally prospective randomized trials designed to address specific issues and indications for surgery.

Several clinical trials were instrumental in establishing CEA as a superior treatment over medical management in the prevention of stroke.

NASCET, or the North American Symptomatic Carotid Endarterectomy trial, enrolled 2885 symptomatic patients who were randomized over 9 years from December 1987 to December 1996. Symptomatic patients were described as those with non-disabling stroke or transient ischemic attack within 180 days prior to enrollment in the study. Patients had varying degrees of carotid stenosis by angiography and lesions were categorized as follows: 1) low, moderate stenosis $(<50 \%), 2)$ moderate stenosis $(50-69 \%)$, and 3$)$ severe stenosis (70-99\%). Patients were assigned to either CEA and best medical management or best medical management alone. The latter entailed antiplatelet therapy (usually aspirin) and risk factor modification if indicated. ${ }^{1}$

In February 1991, approximately 3 years after the trial began; enrollment in the severe stenosis group (70-99\%) was discontinued due to overwhelming evidence in favor of CEA as a superior treatment. The results showed that a cumulative risk of any ipsilateral stroke at two years was $26 \%$ for the medical management patients, and only $9 \%$ for the surgical patients; an absolute difference of $17.0 \pm 3.5 \%$ $(\mathrm{p}<0.001)^{2}$

Further analysis indicated that, while the greatest benefit of CEA was demonstrated in the NASCET patients with severe stenosis, the superiority of CEA over medical therapy was also demonstrated in patients with moderate stenosis (50$69 \%$ ). In this group, the 5 -year rate of ipsilateral stroke was $15.7 \%$ in the surgical group and $22.2 \%$ in the medical group $(\mathrm{p}=0.045)^{1}$ 
Two major trials examined the benefits of CEA in the asymptomatic patient population.

The Asymptomatic Carotid Atherosclerosis Study (ACAS) randomized 1,662 asymptomatic patients with $>60 \%$ stenosis by angiography. Enrollment occurred from December 1987 to December 1993. Patients assigned to the medical management group received aspirin daily as well as risk factor management. After a median follow-up of 2.7 years, it was estimated that the aggregate risk of ipsilateral stroke over 5 years and any perioperative stroke or death was 5.1\% in the CEA group and $11 \%$ in the medical management group. The aggregate risk reduction was $53 \%(\mathrm{p}=0.004){ }^{3}$

The Veterans Affairs Cooperative Study (VACS) enrolled 22 asymptomatic men from 1983 to 1991 who had at least 50\% carotid stenosis by angiography. All patients, regardless of randomization, received $650 \mathrm{mg}$ of ASA twice a day. When neurological endpoints were determined for the entire duration of the trial, patients treated with CEA had an $8 \%$ overall rate of ipsilateral neurological events (TIA, monocular blindness, stroke) compared with $20.6 \%$ for the medical management group $(p<0.001)$. For ipsilateral stroke alone throughout the course of the trial, the rates were $4.7 \%$ for the surgical group and $9.4 \%$ for the medical group, demonstrating a trend, though not statistically significant, towards superiority of CEA. This trial demonstrated that CEA significantly reduced the incidence of neurological events as compared to those treated medically. The data from these randomized trials provided information that assisted an ad hoc committee of the American Heart Association (AHA) in issuing guidelines for endarterectomy in 1995 with an update in $1998 .{ }^{5}$ Recommendations were given for both symptomatic and asymptomatic patients with carotid artery disease. For each indication there is a stated surgical risk that is a combined estimate of the patient's medical fitness for surgery along with the surgeon's risk of morbidity and mortality for the surgical procedure. Surgical risk categories range from 3\% to $10 \%$. Under the current AHA guidelines, recommendations for CEA in the asymptomatic population are generally for high-grade lesions $(>60 \%)$ in patients with relatively good health. For symptomatic patients, CEA is recommended for patients with recent cerebrovascular ischemic event who have at least $50 \%$ stenosis in the carotid artery.

Reports of carotid endarterectomy (CEA) performed in combination with CABG have also increased steadily since Bernhard and colleague's initial report of 16 such cases in 1972. ${ }^{6}$ Coexistence of symptomatic coronary artery disease and significant carotid artery stenosis ranges from $3.4 \%$ to $22 \% .^{7}$ In the past two decades, the high-risk potential for neurologic dysfunction after coronary artery bypass grafting
(CABG) in patients with concomitant carotid stenosis has been a real challenge for surgeons in terms of determining which operative sequence offers the highest freedom from cardiac or cerebral complications. In general, the incidence of postoperative stroke after $\mathrm{CABG}$ ranges from $0.7 \%$ to $5 \%$. 7,8 Coronary revascularization in a patient with internal carotid artery stenosis more than $50 \%$ is associated with a postoperative stroke rate of $6 \%$, which increases significantly to more than $16 \%$ when stenosis is more than $90 \%$. $9-13$ To reduce the potential risk for postoperative stroke after CABG in patients with significant or symptomatic carotid artery stenosis, many surgeons have advocated combined CABG with unilateral carotid endarterectomy. However, clinical experience with the concomitant approach is conflicting. 9,11,14 Biases in patient selection criteria, variations in operative techniques, and intraoperative cerebral protective measures may help to explain a wide range of postoperative stroke rate (from $2 \%$ to $20 \%$ ) associated with the combined approach.

In Canada, $0.51 \%$ of CABG procedures were combined CEACABG. The adjusted stroke and death rate was 2.67-fold greater in the combined CEA-CABG group compared to CABG alone. ${ }^{15}$

Another study in USA, determined hospital mortality and postoperative stroke rates after combined carotid endarterectomy (CEA) and coronary artery bypass graft surgery (CABG) versus CABG alone, using the US Nationwide Inpatient Sample. The mortality rate in CEA-CABG combined was $1.06 \%$. After correcting for comorbidities, the odds ratio for postoperative stroke or death for CEA-CABG was 1.38 (95\% CI 1.27 to 1.50$)$ versus CABG alone. Guided by the increase in postoperative stroke and death, this survey advocated a randomized, controlled clinical trial of combined CEA-CABG. ${ }^{16}$

Though concurrent Carotid Endarterectomy (CEA) and Coronary Artery Bypass Graft (CABG) is associated with an increased incidence of stroke and death compared to isolated $\mathrm{CABG}$, it is unclear whether this reflects two concurrent operative procedures or the increased risk in patients with more extensive atherosclerosis. To address this question a case controlled study was performed using data from the New York State Cardiac Database from 1997-1998. Patients who underwent combined CEA-CABG were compared to all isolated $C A B G$ patients and a risk matched cohort of isolated CABG patients. The 35,539 isolated CABG patients had fewer post operative complications than the 744 combined CEACABG patients, but also had a lower overall risk profile. After propensity matching for confounding variables, no differences in length of stay, stroke, myocardial infarction, 
death or total adverse events were observed. While increased complications are reported after CEA-CABG, these do not differ from those of a risk-matched cohort of isolated CABG patients. Thus the major morbidity of combined CEA-CABG is due to inherent patient risk and not the addition of CEA to CABG. ${ }^{17}$

A significant cost reduction is likely if patients who require coronary artery bypass grafting with significant carotid stenosis have simultaneous carotid endarterectomy and bypass grafting, provided risk is not increased. To investigate this issue, a retrospective analysis was made on identified cases from February 1977 to May 1994 with first-time isolated carotid endarterectomy, coronary bypass, or combined procedures. In the isolated carotid endarterectomy population, median age was 69 years and $58 \%(85 / 146)$ were male, as compared with 68 years and $68 \%(68 / 100)$ male in the combined group; median age of the coronary bypass cohort was 65 years and $76 \%(381 / 500)$ male. A significantly higher percentage of patients in the coronary bypass versus combined group were in New York Heart Association functional class IV. In the combined group there was a significantly higher incidence of older age, diabetes, hypertension, hyperlipidemia renal failure, and congestive heart failure. There was no difference among the three groups with respect to hospital mortality $(0 \%, 3.4 \%$, and $4.0 \%$, respectively) and permanent stroke $(0.7 \%, 1.2 \%$, and $0 \%$, respectively). Hospital costs were $\$ 4,896, \$ 10,959$ and $\$ 11,089$, respectively, with a savings of $\$ 4,766(30 \%)$, and Medicare hospital reimbursement was $\$ 8,575, \$ 23,071$, and $\$ 23,071$, respectively, with a savings of $\$ 10,077$ (25.3\%). Thus, in appropriate patients, a combined procedure is cost effective, eliminating a second surgical procedure cost of the postoperative stay $(3.7 \pm 2.4$ days) associated with isolated carotid endarterectomy. Risk of permanent stroke or death is not increased. ${ }^{18}$

Coronary artery bypass grafting ( $\mathrm{CABG})$ is conventionally performed utilizing cardiopulmonary bypass ( $\mathrm{CPB}$ ), aortic cross clamping, and cardioplegic cardiac arrest. However, CABG without $\mathrm{CPB}$ i.e. off-pump CABG (OP-CABG) is now practiced widely and is an accepted technique of myocardial revascularization. Off-pump CABG eliminates the systemic inflammatory response syndrome (SIRS) associated with CPB and is shown to be associated with significantly less myocardial damage as compared to CABG with $\mathrm{CPB}$. A combined CEA and CABG performed as a one-stage procedure offers better overall outcome than a two-stage approach. Traditionally, cerebral perfusion during CEA is maintained by the use of shunts inserted into the proximal and distal carotid arteries. An alternative method of maintaining carotid perfusion during combined carotid endarterectomy and off-pump coronary artery bypass grafting involves insertion of a cannula in the ascending aorta after a median sternotomy. This cannula is connected to a perfusion cannula, the distal end of which is inserted into the carotid artery beyond the carotid arteriotomy. This technique of aorticocarotid shunting and carotid perfusion was utilized in nine patients who underwent successful combined carotid endarterectomy and off-pump coronary artery bypass grafting. ${ }^{19}$

A retrospective nonrandomized chart review was performed in 33 patients with unstable angina and bilateral carotid artery stenosis, more than $70 \%$, undergoing simultaneous coronary artery bypass grafting and bilateral carotid endarterectomy using an eversion technique. Concomitant coronary artery bypass grafting with bilateral carotid endarterectomy was performed urgently in $24(73 \%)$ and electively in $9(27 \%)$ patients. The average carotid artery cross-clamp and total perfusion times were $14.7 \pm 4.9 \mathrm{~min}-$ utes and $123 \pm 29.2$ minutes, respectively. The average length of stay in the cardiopulmonary intensive care unit was $4.2 \pm 14.2$ days and total hospital stay was $16.2 \pm 20.5$ days. Postoperative in-hospital stay was $14.9 \pm 20.3$ days. There were no postoperative strokes. Twenty-one (64\%) patients were discharged before the tenth postoperative day. Nonfatal postoperative complications occurred in $27 \%$ (9 of 33 ) of patients. The overall 30 -day mortality was $6.1 \%$ ( 2 of 33 ) and that was unrelated to primary cardiac or cerebrovascular events. Favorable outcome supports the justification for performing concomitant coronary artery bypass grafting with bilateral carotid endarterectomies in selected patients. ${ }^{20}$

Another study involving 340 patients who underwent simultaneous carotid endarterectomy and myocardial revascularization from February 1985 to September 1998 was reviwed. The average age of the patients was 65.3 years; $45.6 \%$ were neurologically symptomatic, and $44.4 \%$ had bilateral carotid stenosis. The indication for carotid endarterectomy was lumen diameter reduction of more than $75 \%$, angiographic signs of thrombogenic endovascular morphology, or both. Carotid endarterectomy was performed in conjunction with cardiopulmonary bypass with mild hypothermia, hemodilution, systemic heparinization, and controlled hemodynamics under pulsatile perfusion for additional cerebral protection. There were 16 perioperative neurologic complications (4.7\%), 11 permanent deficits (3.2\%), and 9 cardiac complications $(2.6 \%$ ). Early mortality was $2.6 \%$ (SE 0.8\%): 2 patients had a stroke and 2 had a myocardial infarction. The 5-year survival was $78.9 \%$ (SE 2.6\%), and freedom from ipsilateral stroke and cardiac event were 93.2\% (SE $1.5 \%$ ) and $87.5 \%$ (SE 2.1\%), respectively. The 
predictor for early death was age over 70 years, and predictors for late death were age over 70 years, previous myocardial infarction, previous stroke, and bilateral carotid stenosis of greater than $90 \%$. On the basis of the long-term results, it is estimated that simultaneous carotid endarterectomy and myocardial revascularization in conjunction with cardiopulmonary bypass is a method safe enough to prefer its routine use with acceptable low operative risk and satisfactory long-term morbidity. ${ }^{21}$

\section{Conclusion}

Various studies proved that combined carotid endarterectomy and coronary artery bypass grafting is a cost effective and safe procedure and does not increase overall morbidity and mortality and can be used in selected group of patients.

\section{References}

1. Barnett, H.J.M., D.W. Taylor, et al. Benefit of Carotid Endarterectomy in Patients with Symptomatic Moderate or Severe Stenosis. N Engl J Med 1998; 339:1415-25

2. NASCET Collaborators. Beneficial Effect of Carotid Endarterectomy in Symptomatic Patients with High Grade Carotid Stenosis. N Engl J Med 1991; 325:445-53

3. Executive Committee for the Asymptomatic Carotid Atherosclerosis Study. Endarterectomy for Asymptomatic Carotid Artery Stenosis. JAMA 1995; 273: 1421-28

4. Hobson, R.W., II, D.G. Weiss, et al. Efficacy of Carotid Endarterectomy for Asymptomatic Carotid Stenosis. N Engl J Med 1993; 328: 221-27

5. Moore, W.S., H. J. M. Barnett et al. Guidelines for Carotid Endarterectomy - A Multi-disciplinary Consensus Statement from the Ad Hoc Committee, American Heart Association. Stroke 1995; 26:188-201

6. Bernhard VM, Johnson WD, Peterson JJ. Carotid artery stenosis. Association with surgery for coronary artery disease. Arch Surg 1972; 105: 837-40

7. Mackey W.C., Khabbaz K., Bojar R., et al. Simultaneous carotid endarterectomy and coronary bypass: perioperative risk and long-term survival. J Vasc Surg 1996;24:58-64

8. Hertzer N.R., Loop F.D., Taylor P.C., et al. Combined myocardial revascularization and carotid endarterectomy. $J$ Thorac Cardiovasc Surg 1983;85:577-89

9. Breslau P.J., Fell G., Ivey T.D., et al. Carotid arterial disease in patients undergoing coronary artery bypass operations. $J$ Thorac Cardiovasc Surg 1981;82:765-67

10. Faggioli G.L., Curl G.R., Ricotta J.J., et al. The role of carotid screening before coronary artery bypass. J Vasc Surg 1990;12:724-31
11. Rizzo R.J., Whittemore A.D., Couper G.S., et al. Combined carotid and coronary revascularization: the preferred approach to the severe vasculopath. Ann Thorac Surg 1992;54:1099-1109

12. Chang B.B., Darling R.C., III, Shah D.M., et al. Carotid endarterectomy can be safely performed with acceptable mortality and morbidity in patients requiring coronary artery bypass grafts. Am J Surg 1994;168:94-96

13. Brener B.J., Brief D.K., Alpert J., et al. The risk of stroke in patients with asymptomatic carotid stenosis undergoing cardiac surgery: a follow-up study. J Vasc Surg 1987;5:269-77.

14. Furlan A.J., Cracium A.R. Risk of stroke during coronary artery bypass graft surgery in patients with internal carotid artery disease documented by angiography. Stroke 1985;16:797-99

15. M. D. Hill, F. M. Shrive, J. Kennedy, T. E. Feasby, and W. A. Ghali. Simultaneous carotid endarterectomy and coronary artery bypass surgery in Canada . NEUROLOGY 2005; 64: 1435-37

16. Richard M. Dubinsky, Sue Min Lai. Mortality from combined carotid endarterectomy and coronary artery bypass surgery in the US . NEUROLOGY 2007; 68:195 -97

17. L. Philipp Wall1, Eugene Blackstone2, John Ricotta11SUNY. The Influence Of Concurrent Carotid Endarterectomy On Coronary Bypass: A Case Controlled Study . New England Society for Vascular Surgery, Cleveland Clinic Foundation, News, July 14, 2004

18. Pat O. Daily, Richard K. Freeman, Walter P. Dembitsky, et al. Cost reduction by combined carotid endarterectomy and coronary artery bypass grafting. J Thorac Cardiovasc Surg 1996;111:1185-93

19. Parachuri V Rao, Praveen K Hosabettu, Sanjay Dhaded et al. Distal Carotid Perfusion in Combined Carotid Endarterectomy and OP-CABG. Asian Cardiovasc Thorac Ann 2007;15:164-66

20. Mark Dylewski, Charles C. Canver, Jyotirmay Chanda et al. Coronary artery bypass combined with bilateral carotid endarterectomy . Ann Thorac Surg 2001;71:777-81

21. Kazutomo Minami, Kazuaki Fukahara, Dietmar Boethig et al. Long-term results of simultaneous carotid endarterectomy and myocardial revascularization with cardiopulmonary bypass used for both procedures. J Thorac Cardiovasc Surg 2000;119:764-72 